Comunicação Breve

Brief Communication

Lucas Cainan Paulino ${ }^{1}$ (D)

Marcia Simões-Zenari' ${ }^{1}$

Katia Nemr ${ }^{1}$ (1)

Descritores

Voz

Distúrbios da Voz

Exposição Ocupacional

Saúde do Trabalhador

Inquéritos e Questionários

Keywords

Voice

Voice Disorders

Occupational Exposure

Occupational Health

Surveys and Questionnaires
Endereço para correspondência: Marcia Simões-Zenari Faculdade de Medicina, Universidade de São Paulo - USP Rua Cipotânea, 51, Cidade Universitária, São Paulo (SP), Brasil, CEP: 05360-000.

E-mail:marciasz@usp.br

Recebido em: Abril 15, 2019

\section{Protocolo de Rastreio do Risco de Disfonia para Atores de Teatro Musical: resultados preliminares}

\section{Dysphonia Risk Screening Protocol for Musical Theatre Actors: a preliminary study}

\section{RESUMO}

Objetivo: Propor o Protocolo de Rastreio do Risco de Disfonia para Atores do Teatro Musical (PRRD-TM), verificar sua aplicabilidade em associação ao Protocolo de Rastreio do Risco de Disfonia Geral (PRRD-G), correlacionar escores finais de ambos, e desses com o escore total, e comparar o risco de disfonia entre atores com e sem queixa vocal. Método: Estudo transversal observacional com 34 atores de teatro musical adultos, ambos os sexos, com e sem queixa vocal, profissionais ou estudantes. Os questionários foram aplicados individualmente. A análise estatística possibilitou verificar a correlação entre os escores de risco de disfonia e para comparação entre os grupos com e sem queixa vocal. Resultados: A maioria dos participantes era do gênero masculino, jovens adultos, atores profissionais e sem queixa vocal. Observou-se elevado risco de disfonia, evidenciado pela aplicação do PRRD-G, com escores médios compatíveis com valores encontrados em indivíduos com disfonia, e reforçado pelos índices encontrados com aplicação do PRRD-TM. Observou-se correlação moderada e diretamente proporcional entre os escores dos dois questionários e desses com o escore total. Escores mais elevados do PRRD-G foram encontrados no grupo que apresentou queixa vocal. Conclusão: O PRRD-TM mostrou-se viável e de fácil aplicabilidade e apresentou correlação positiva com o escore do PRRD-G e com o escore total. Elevado risco de disfonia foi evidenciado em indivíduos com queixa vocal. Apesar do escore específico do PRRD-TM não diferenciar atores de teatro musical com e sem queixa vocal, tanto o escore do PRRD-G quanto o escore total realizaram tal diferenciação.

\begin{abstract}
Purpose: To propose the Dysphonia Risk Screening Protocol for Musical Theatre Actors (DRSP-MTA), to verify its applicability in association with the General Dysphonia Risk Screening Protocol (G-DRSP), to correlate the final scores of both, and these with the total risk score, and to compare the risk of dysphonia measured in musical theater actors with and without vocal complaint. Methods: An observational cross-sectional study with 34 musical theater actors, adults, of both genders, with and without vocal complaints and regardless of whether they are professionals or students. The questionnaires were applied individually. Statistical analysis made it possible to verify the correlation between the dysphonia risk scores and to compare the groups with and without vocal complaint. Results: Most of the participants were male, young adults, professional actors and without vocal complaint. There was a high risk of dysphonia, evidenced by the application of G-DRSP, with means scores compatible with values found in individuals with dysphonia, and reinforced by the indices found with DRSP-MTA application. There was a moderate and directly proportional correlation between the two questionnaire scores; and a correlation of both with the total risk score. Higher G-DRSP scores were observed in the vocal complaint group. Conclusion: DRSP-MTA was feasible and easy to apply and was positively correlated with the total score and G-DRSP score. A high risk of dysphonia was evidenced in individuals with vocal complaints. Although the specific DRSP-MTA score did not differentiate musical theatre actors with and without vocal complaints, the G-DRSP score and the total risk score performed such differentiation.
\end{abstract}

Trabalho realizado no Departamento de Fisioterapia, Fonoaudiologia e Terapia Ocupacional, Faculdade de Medicina, Universidade de São Paulo - USP - São Paulo (SP), Brasil.

${ }^{1}$ Faculdade de Medicina, Universidade de São Paulo - USP - São Paulo (SP), Brasil.

Fonte de financiamento: Bolsa de Iniciação Científica PIBIC, Conselho Nacional de Desenvolvimento Científico e Tecnológico (CNPq), processo ${ }^{\circ}$ 146636/2016-3.

Conflito de interesses: nada a declarar. 


\section{INTRODUÇÃO}

O uso de protocolos padronizados e validados favorece a prática fonoaudiológica na medida em que facilita a comparação dos resultados entre diferentes serviços e permite a realização de estudos que direcionem e aperfeiçoem procedimentos, o que contribui para a prática baseada em evidências e proporciona melhor qualidade de atendimento ao paciente ${ }^{(1-4)}$.

Instrumento destinado à investigação inicial em voz denominado Protocolo de Rastreio de Risco de Disfonia - Geral (PRRD-G), com cálculo de escores, apresentou elevada sensibilidade para diferenciar grupos com e sem disfonia e demonstrou efetividade na clínica de $\mathrm{voz}^{(4)}$. O mesmo instrumento foi aplicado junto a indivíduos com diferentes diagnósticos laríngeos e seu escore correlacionou-se positivamente com o grau de alteração vocal ${ }^{(5)}$.

O PRRD-G tem sua aplicabilidade indicada a indivíduos de qualquer faixa etária, gênero e independentemente do uso profissional da voz, permitindo o estudo da multiplicidade de fatores envolvidos no desenvolvimento da disfonia ${ }^{(4)}$. A proposta é que analise fatores gerais relacionados à voz, sendo necessário complementá-lo com instrumentos específicos de acordo com a faixa etária e profissão. O Protocolo de Rastreio de Risco de Disfonia Específico para Professores (PRRD-Pro), por exemplo, demonstrou que seu escore, associado ao escore do PRRD-G, sustenta a diferenciação entre grupos com e sem alteração vocal, além de fornecer dados qualitativos relevantes para avaliação e planejamento do atendimento fonoaudiológico junto a esta categoria profissional em especial ${ }^{(6)}$.

Uma variável possível de ser extraída pela aplicação do PRRD-G associado a um protocolo de rastreio do risco de disfonia específico é o escore total do risco de disfonia. Este escore é representado pela soma simples do escore do PRRDGeral e do escore do PRRD-Específico. Teoricamente esse escore total possibilita a quantificação do risco de disfonia de maneira condizente com a realidade do indivíduo avaliado, pois permite mensuração única, tanto dos riscos gerais, aos quais toda a população pode estar exposta independentemente de faixa etária e profissão, quanto dos riscos específicos relacionados a diferentes faixas etárias ou profissões.

No que se refere à voz de atores do teatro musical há muitas especificidades a serem consideradas. Constituem um grupo especial dentro da elite vocal devido à necessidade de cantar, atuar e dançar ${ }^{(7)}$, e são considerados mais suscetíveis para o desenvolvimento de problemas vocais ${ }^{(8)}$. Na avaliação fonoaudiológica desses profissionais, além dos aspectos relacionados à produção vocal, é necessário compreender as demandas específicas de cada personagem, as possíveis condições adversas em relação ao ambiente, o preparo do ator e o estilo musical da produção. É importante considerar a vasta gama de estilos musicais existentes neste cenário e as diferenças entre eles ${ }^{(9-11)}$. Além disso, destaca-se a estrutura da produção de teatro musical que pode envolver cenografias grandiosas e longas coreografias, o que exige controle preciso tanto das técnicas vocais (ajustes laríngeos, do trato vocal e de suporte respiratório) quanto expressivos ${ }^{(7)}$.

A necessidade de combinar canto projetado e dança atlética, que é a base do teatro musical, numa jornada de muitos ensaios e apresentações, pressupõe efeitos fisiológicos que precisam ser melhor conhecidos não apenas pela estética do musical, mas principalmente pelo bem-estar do artista e longevidade de sua carreira ${ }^{(12)}$.

No entanto, ainda são limitados os dados referentes aos hábitos e sintomas vocais e qualidade de voz desta população ${ }^{(7)}$.

Um instrumento de investigação específico para ser utilizado junto aos atores do teatro musical contribuirá para compreensão mais aprofundada dos riscos e de aspectos que possam interferir em sua performance vocal.

O presente estudo teve por objetivo propor o Protocolo de Rastreio do Risco de Disfonia para Atores do Teatro Musical (PRRD-TM), verificar sua aplicabilidade, correlacionar seu escore específico ao escore do Protocolo de Rastreio do Risco de Disfonia Geral (PRRD-G) e com o escore total de risco de disfonia e, por fim, comparar o risco de disfonia mensurado em atores de teatro musical com e sem queixa de voz.

\section{MÉTODO}

Estudo transversal observacional aprovado pelo Comitê de Ética da Faculdade de Medicina da Universidade de São Paulo $\left(n^{\circ} 0560 / 10\right)$.

A amostra formada por conveniência foi composta por 44 atores de teatro musical com idades entre 18 e 47 anos (média de 25,7 anos; $\pm 6,08$ ), dos quais 28 eram homens ( 17 profissionais e 11 estudantes) e 16 eram mulheres (8 profissionais e 8 estudantes).

Inicialmente foi perguntado a cada participante: "Você tem alguma queixa relacionada à sua voz?". Dezessete (39\%) responderam afirmativamente e $27(70 \%)$ relataram não ter queixa vocal. Foram estabelecidos, assim, dois grupos: com queixa vocal (CQV) e sem queixa vocal (SQV).

O grupo CQV foi composto por: dez participantes homens $(58,8 \%)$ e sete mulheres $(41,2 \%)$, sendo onze estudantes $(64,7 \%)$ e seis profissionais $(35,3 \%)$, totalizando 17 indivíduos (média de idade de 23,7 anos $\pm 4,01$ ); o grupo SQV foi composto por dezoito participantes homens $(66,7 \%)$ e nove mulheres $(33,3 \%)$ sendo oito estudantes $(29,6 \%)$ e dezenove profissionais $(70,4 \%)$ totalizando 27 participantes (média de idade de 27 anos $\pm 6,86$ ).

Todos os indivíduos preencheram o Termo de Consentimento Livre e Esclarecido e responderam ao PRRD- $\mathrm{G}^{(4)}$ e ao PRRD-TM, sendo este último elaborado para o presente estudo (Apêndice A). Os questionários foram aplicados pelo mesmo pesquisador, individualmente, em local silencioso e da conveniência dos participantes.

Para a elaboração do PRRD-TM foi realizada ampla revisão da literatura sobre o tema e duas aplicações piloto foram efetuadas para os ajustes necessários. Os dados das aplicações piloto não foram incluídos neste estudo.

Tanto o PRRD-G quanto o PRRD-TM possibilitam cálculo de escores parciais e um escore total. O escore do PRRD-G pode variar de zero a 131 pontos e o do PRRD-TM de zero a 166 pontos. Em ambos, quanto mais negativa a resposta mais elevada é a pontuação e maior o risco. Para o PRRD-G a pontuação de corte para risco elevado de disfonia foi definida, sendo 22,75 para homens adultos e 29,25 para mulheres adultas ${ }^{(4)}$. 
O PRRD-TM é composto por 28 perguntas, das quais quatro são qualitativas e 24 pontuadas em subescores (apresentando subitens), são eles: momento da carreira, atuação em outras profissões, formação artística, aula de canto, aula de dança, preparação física, ensaios, uso de microfone, condições ambientais, tipo respiratório autorreferido, dificuldades para manter a qualidade vocal, função na produção, qualidade vocal antes da prática artística, qualidade vocal depois da prática artística, estresse, aquecimento e desaquecimento vocais, uso vocal diário, fumo, ingestão de bebida alcoólica, uso de drogas, uso de prótese dentária, questões específicas para as mulheres, sinais e sintomas.

Para este estudo foram considerados os dois escores totais, além de ter sido criado um escore final de risco de disfonia (ER) a partir da soma simples entre ambos (PRRD-G + PRRD-TM; variação de zero a 298).

A análise dos dados foi descritiva e inferencial. Para definição da análise estatística foi aplicado o teste de normalidade de Kolmogorov-Smirnov. Para análise de correlação entre os escores finais dos dois instrumentos aplicados foi usado o teste de Correlação de Pearson, com interpretação do coeficiente de correlação baseada em Dancey e Reidy ${ }^{(12)}$ que apontam como fraca a correlação com coeficiente de 0,1 a 0,3 , moderada de 0,4 a 0,6 e forte de 0,7 a 1; para comparação dos escores entre os grupos CQV e SQV foi utilizado o teste t de Student. O nível de significância foi estabelecido em 5\%.

\section{RESULTADOS}

$\mathrm{Na}$ amostra de 44 atores observaram-se médias de 40,58 $( \pm 15,48)$ no PRRD-G, $58,39( \pm 15,80)$ no PRRD-TM e 98,98 $( \pm 26,86)$ no escore total. Os valores obtidos em cada grupo foram: $\mathrm{CQV}=51,12( \pm 17,26)$ no PRRD-G, 60,82 $( \pm 17,38)$ no PRRD-TM e $111,95( \pm 30,38)$ no escore total (PRRD-G + PRRD-TM); $\mathrm{SQV}=33,95( \pm 9,74)$ no PRRD-G, 56,86 $( \pm 14,85)$ no PRRD-TM e $90,82( \pm 21,13)$ no ER.

O escore do PRRD-TM apresentou correlação positiva moderada com o escore do PRRD-G, tanto no grupo CQV quanto no SQV. Também se correlacionou positivamente com o ER em ambos os grupos sendo uma correlação forte em ambos os grupos SQV e CQV, o mesmo ocorreu com o escore do PRRD-G que apresentou correlação positiva e forte com o ER em ambos os grupos (Tabela 1).

O ER e o escore do PRRD-G diferenciaram atores de teatro musical com e sem queixa vocal, identificando-se médias mais elevadas no grupo CQV para ambos os escores (Tabela 2).

\section{DISCUSSÃO}

Ao se considerar os pontos de corte para baixo e alto risco de disfonia de referência ${ }^{(6)}$ evidenciou-se elevado risco de disfonia em atores de teatro musical com queixa de alteração de voz. Além disso, o valores do escore PRRD-G observados estão próximos à média encontrada em indivíduos com disfonia $^{(6)}$. Os achados laringológicos ${ }^{(7)}$, a carga vocal elevada ${ }^{(8)}$ e os resultados do presente estudo reforçam a necessidade de pesquisas com essa população, identificando e descrevendo suas necessidades e os riscos, tanto em estudantes quanto em profissionais. Além de explicitar a necessidade de programas específicos de treinamento ${ }^{(13)}$.

Os atores de teatro musical têm elevada demanda vocal e também grande exigência quanto à qualidade vocal ${ }^{(14)}$. Assim, alterações vocais, ainda que de grau discreto, podem impactar fortemente em suas carreiras. A excelência na execução de ajustes vocais específicos para cada personagem e em cada produção artística lhes é exigida; tanto ajustes relacionados à fonte glótica quanto relativos ao trato vocal e respiração ${ }^{(13)}$. Além disso, existe a elevada carga vocal composta pelo volume de apresentações semanais, ensaios e coreografias, muitas vezes complexas e associadas ao uso da voz $z^{(7)}$. Qualquer problema, seja vocal, físico ou emocional, poderá comprometer a performance do ator e resultar em dificuldades para que se estabeleça no mercado de trabalho ${ }^{(7,8,10)}$.

Independentemente da presença ou não de queixa vocal, os escores médios obtidos com a aplicação dos protocolos de rastreio indicaram risco elevado de disfonia ${ }^{(4)}$. O valor médio obtido no PRRD-G, além de estar acima do ponto de corte para risco elevado de disfonia ${ }^{(4)}$, está próximo à média encontrada em indivíduos que apresentaram disfonia ${ }^{(4)}$. Os achados laringológicos ${ }^{(7)}$, a carga vocal elevada ${ }^{(8)} \mathrm{e}$ os resultados do presente estudo reforçam a necessidade de pesquisas com essa população, identificando e descrevendo suas necessidades e os riscos, tanto em estudantes quanto em profissionais. Além de explicitar a necessidade de programas específicos de treinamento ${ }^{(13)}$.

Nesse contexto, o PRRD-TM apresentou-se inovador em sua proposta e, além de trazer questões quantitativas, oferece dados qualitativos pertinentes ao rastreio do risco de disfonia em atores do teatro musical.

As correlações positivas entre os escores do PRRD-TM e do PRRD-G, tanto no grupo com queixa vocal quanto no grupo sem queixa, reforçam a importância de se associar ambos questionários na investigação do risco de disfonia nessa população ${ }^{(6)}$. Quanto mais presentes os riscos gerais mais presentes estarão os riscos específicos referidos pelo ator de teatro musical. Essa evidência também é reforçada pelas correlações positivas observadas entre o escore total e os escores do PRRD-G e do PRRD-TM, observadas também em ambos os grupos (CQV e SQV).

A presença de queixa vocal foi determinante para maior risco de disfonia evidenciado pelo PRRD-G e pelo escore total. Os subescores disponíveis neste questionário poderão ser analisados futuramente para detalhar essa diferença. Pesquisa anterior mostrou eficiência do questionário na diferenciação de indivíduos com e sem disfonia ${ }^{(4)}$.

$\mathrm{O}$ escore do PRRD-TM não diferenciou os grupos CQV e SQV. Questionar o ator sobre queixa relacionada à voz pode suscitar diversas interpretações, pois para uns poderá refletir dificuldades em ajustes específicos de fala e canto ${ }^{(7)}$. Para outros pode se relacionar à presença de alterações na qualidade vocal, por exemplo. $\mathrm{O}$ achado da presente pesquisa pode ser indicativo de que, neste caso, a queixa vocal estaria mais relacionada a aspectos gerais como sinais e sintomas vocais, comorbidades e alterações vocais anteriores, que poderão ser explorados na continuidade do estudo nessa temática. 
Tabela 1. Análise de correlação entre os escores dos questionários por grupo com e sem queixa vocal

\begin{tabular}{|c|c|c|c|c|c|}
\hline \multirow{2}{*}{ Grupos } & \multirow{2}{*}{ Escores } & & \multicolumn{3}{|c|}{ Escores } \\
\hline & & & PRRD-TM & PRRD-G & Total \\
\hline \multirow{4}{*}{ Com Queixa Vocal } & \multirow{2}{*}{ PRRD-TM } & Correlação de Pearson & 1,000 & ,537 & ,878 \\
\hline & & $\mathrm{n}$ & 17 & 17 & 17 \\
\hline & \multirow[b]{2}{*}{ PRRD-G } & Correlação de Pearson &, 537 & 1,000 & ,876 \\
\hline & & Significância &, $026^{*}$ & &, $000^{*}$ \\
\hline \multirow{5}{*}{ Sem Queixa Vocal } & \multirow[t]{2}{*}{ PRRD-TM } & Significância & &, $018^{\star}$ &, $000^{*}$ \\
\hline & & $\mathrm{n}$ & 17 & 27 & 27 \\
\hline & \multirow{3}{*}{ PRRD-G } & Correlação de Pearson & ,453 & 1,000 &, 779 \\
\hline & & Significância &, $018^{*}$ & &, $000^{*}$ \\
\hline & & $\mathrm{n}$ & 27 & 27 & 27 \\
\hline
\end{tabular}

Teste de correlação de Pearson *Estatisticamente significativo

Legenda: PRRD-TM = Protocolo de Rastreio de Risco de Disfonia-Teatro Musical; PRRD-G = Protocolo de Rastreio de Risco de Disfonia-Geral

Tabela 2. Comparação entre os grupos com e sem queixa vocal em relação aos escores dos questionários

\begin{tabular}{|c|c|c|c|c|}
\hline \multirow{2}{*}{\multicolumn{2}{|c|}{ Escores }} & \multicolumn{2}{|c|}{ Grupos } & \multirow[t]{2}{*}{ valor de $p$} \\
\hline & & Com queixa vocal & Sem queixa vocal & \\
\hline & Média & 60,82 & 56,86 & \\
\hline \multirow[t]{3}{*}{ PRRD-TM } & Desvio-padrão & 17,38 & 14,85 & 0,424 \\
\hline & $\mathrm{n}$ & 17 & 27 & \\
\hline & Média & 51,12 & 33,95 & \\
\hline \multirow[t]{3}{*}{ PRRD-G } & Desvio-padrão & 17,26 & 9,74 & $0,001^{*}$ \\
\hline & $\mathrm{n}$ & 17 & 27 & \\
\hline & Média & 111,95 & 90,82 & \\
\hline \multirow[t]{2}{*}{ Total } & Desvio-padrão & 30,38 & 21,13 & $0,009^{*}$ \\
\hline & $\mathrm{n}$ & 17 & 27 & \\
\hline
\end{tabular}

Teste t-Student *Estatisticamente significativo

Legenda: PRRD-TM = Protocolo de Rastreio de Risco de Disfonia-Teatro Musical; PRRD-G = Protocolo de Rastreio de Risco de Disfonia-Geral

Outro estudo ${ }^{(7)}$ encontrou alta prevalência de sinais e sintomas vocais negativos em 31 estudantes de teatro musical como fadiga vocal, garganta seca e desconforto no trato vocal, mas que não foram associados à presença de alteração vocal. Ainda assim, alta ocorrência de alterações laringológicas foi encontrada, como lesões inflamatórias, e que também podem explicar, pelo menos em parte, alguns sinais e sintomas referidos ${ }^{(7)}$. O sintoma mais mencionado em outro estudo foi também a fadiga vocal, nesse caso nem sempre considerada pelos atores como algo negativo, mas consequência do esforço/dedicação excessivos ${ }^{(8)}$. Indicaram também soprosidade, dificuldade para alcançar notas elevadas e desconforto na garganta ${ }^{(8)}$.

A necessidade de treinamento específico foi destacada em estudo que demonstrou dificuldades respiratórias quando o canto e a dança ocorrem simultaneamente no teatro musical, o que pode levar a sobrecargas, desconfortos e perda da qualidade estética $^{(13)}$.

Os atores de teatro musical com queixa vocal apresentaram maior ER do que aqueles sem queixa vocal, riscos gerais e específicos. Tal achado confirma a hipótese da aplicabilidade dessa variável e indica a necessidade de estudos que a englobem.

Dentre os atores com queixa vocal muitos eram estudantes. Há autores que acreditam que os atores profissionais dominam melhor as técnicas vocais para o canto no teatro musical e por isso teriam risco um pouco menor do que os estudantes ${ }^{(11)}$. Por outro lado, os profissionais podem estar envolvidos em produções teatrais mais exaustivas quanto à performance exigida, número de ensaios e de apresentações e ambiente inadequado, o que pode ainda interferir no tempo para descanso adequado e pouca dedicação aos aspectos de bem-estar vocal como práticas de aquecimento e desaquecimento $\operatorname{vocal}^{(7)}$.

Outro aspecto importante a ser considerado nesta população é a presença de comorbidades que podem interferir na voz. Estudo com estudantes de teatro musical encontrou elevada prevalência de ansiedade ou estresse ${ }^{(7)}$.

Os demais dados coletados com aplicação dos dois questionários envolvem o levantamento de sinais e sintomas, rotina e ambiente de trabalho, ensaios e apresentações, além daqueles relativos à hidratação, fumo e comorbidades, entre outros. E todos serão analisados na continuidade deste estudo, em associação a dados da avaliação da voz, com vistas a ampliar a análise, inclusive quanto à relação entre queixa de disfonia e presença de alteração vocal, e possíveis associações entre presença de queixa e/ou disfonia, escores de risco geral e específico e alterações laríngeas.

Além disso, o impacto de carga vocal tão intensa na função vocal, necessária nesse tipo de atuação, precisa ser melhor conhecido para que se possa prevenir ou ao menos reduzir o dano ${ }^{(8)}$. Os professores 
de teatro musical preocupam-se com os riscos dos estilos de canto por serem altamente desgastantes, física e vocalmente, sendo imprescindível que o ensino ocorra de maneira eficiente e segura ${ }^{(15)}$.

Ampliar o conhecimento qualitativo e quantitativo específico sobre os atores do teatro musical contribuirá com o desenvolvimento de práticas mais direcionadas e efetivas para esta população.

\section{CONCLUSÃO}

Os resultados preliminares do presente estudo indicam que o PRRD-TM proposto possibilitou o levantamento qualitativo e quantitativo de informações específicas sobre atuação no teatro musical, sendo de fácil aplicação e interpretação junto a esta população.

Os atores apresentaram elevado risco de disfonia pelo PRRD-G e pelo PRRD-TM e houve correlação entre os escores dos dois questionários, sugerindo a aplicação associada de ambos.

O escore do PRRD-G e o escore total foram mais elevados entre aqueles com queixa vocal. No grupo com queixa vocal houve presença expressiva de estudantes.

\section{AGRADECIMENTOS}

Ao Conselho Conselho Nacional de Desenvolvimento Científico e Tecnológico (CNPq) e a todos os artistas participantes do estudo que gentil e pacientemente colaboraram para o avanço da ciência.

\section{REFERÊNCIAS}

1. Hazlett DE, Duffy OM, Moorhead SA. Review of the impact of voice training on the vocal quality of professional voice users: implications for vocal health and recommendations for further research. J Voice. 2011;25(2):181-91. http://dx.doi.org/10.1016/j.jvoice.2009.08.005. PMid:20137890.

2. Putnoki DS, Hara F, Oliveira G, Behlau M. Voice-related quality of life: the impact of a dysphonia according to gender, age and occupational use of voice. Rev Soc Bras Fonoaudiol. 2010;15(4):485-90. http://dx.doi. org/10.1590/S1516-80342010000400003.

3. Paoliello K, Oliveira G, Behlau M. Singing voice handicap mapped by different self-assessment instruments. CoDAS. 2013;25(5):463-8. http:// dx.doi.org/10.1590/S2317-17822013005000008. PMid:24408551.

4. Nemr K, Simões-Zenari M, Duarte JMT, Lobrigate KE, Bagatini FA. Dysphonia risk screening protocol. Clinics. 2016;71(3):114-27. http:// dx.doi.org/10.6061/clinics/2016(03)01. PMid:27074171.
5. Nemr K, Cota A, Tsuji D, Simões-Zenari M. Voice deviation, dysphonia risk screening and quality of life in individuals with various laryngeal diagnoses. Clinics (São Paulo). 2018;73:e174. http://dx.doi.org/10.6061/ clinics/2018/e174. PMid:29538494.

6. Silva BG, Chammas TV, Zenari MS, Moreira RR, Samelli AG, Nemr $\mathrm{K}$. Analysis of possible factors of vocal interference during the teaching activity. Rev Saude Publica. 2017;51:124. http://dx.doi.org/10.11606/ S1518-8787.2017051000092. PMid:29236878.

7. D'haeseleer E, Claeys S, Meerschman I, Bettens H, Degeest S, Dijckmans $\mathrm{C}$, et al. Vocal characteristics and laryngoscopic findings in future musical theater performers. J Voice. 2017;31(4):462-9. http://dx.doi.org/10.1016/j. jvoice.2016.11.018. PMid:28081916.

8. Phyland DJ, Thibeault SL, Benninger MS, Vallance N, Greenwood KM, Smith JA. Perspectives on the impact on vocal function of heavy vocal load among working professional music theater performers. J Voice. 2013;27(3):390.e31-9. http://dx.doi.org/10.1016/j.jvoice.2012.12.003. PMid:23415149.

9. Gonçalves A, Elisabeth A, Behlau M. Overall voice and strain level analysis in rock singers. Pro Fono. 2010;22(3):195-200. PMid:21103705.

10. Green K, Freeman W, Edwards M, Meyer D. Trends in Musical Theatre Voice: an analysis of audition requirements for singers. J Voice. 2014;28(3):324-7. http://dx.doi.org/10.1016/j.jvoice.2013.10.007. PMid:24467880.

11. Maxfield L, Manternach B. Perceptual differences between novice and professional music theater singers. J Voice. 2018;32(5):572-7. http://dx.doi. org/10.1016/j.jvoice.2017.08.007. PMid:28888666.

12. Dancey C, Reidy J. Estatística sem matemática para psicologia: usando SPSS para Windows. Porto Alegre: Artmed; 2006.

13. Sliiden T, Beck S, MacDonald I. An evaluation of the breathing strategies and maximum phonation time in musical theater performers during controlled performance tasks. J Voice. 2017;31(2):253.e1-11. http://dx.doi. org/10.1016/j.jvoice.2016.06.025. PMid:27666651.

14. Vilkman E. Voice problems at work: a challenge for occupational safety and health arrangement. Folia Phoniatr Logop. 2000;52(1-3):120-5. http:// dx.doi.org/10.1159/000021519. PMid:10474011.

15. Bourne T, Kenny D. Vocal qualities in music theather voice: perceptions of expert pedagogues. J Voice. 2016;30(1):128.e1-12. http://dx.doi. org/10.1016/j.jvoice.2015.03.008. PMid:25882989.

\section{Contribuição dos autores}

LCP foi responsável pela concepção e desenho do estudo, seleção dos participantes, coleta, análise e interpretação dos dados, escrita e revisão do manuscrito e aprovação da versão final; MSZ foi responsável pela concepção e desenho do estudo, participação na análise dos dados, participação na escrita do manuscrito, revisão do manuscrito e aprovação da versão final; $K N$ foi responsável pela concepção e desenho do estudo, participação na análise dos dados, revisão do manuscrito e aprovação da versão final. 
Apêndice A. Protocolo de Rastreio do Risco de Disfonia Específico para Atores de Teatro Musical (PRRD-TM)

Nome do paciente:

Nome Artístico:

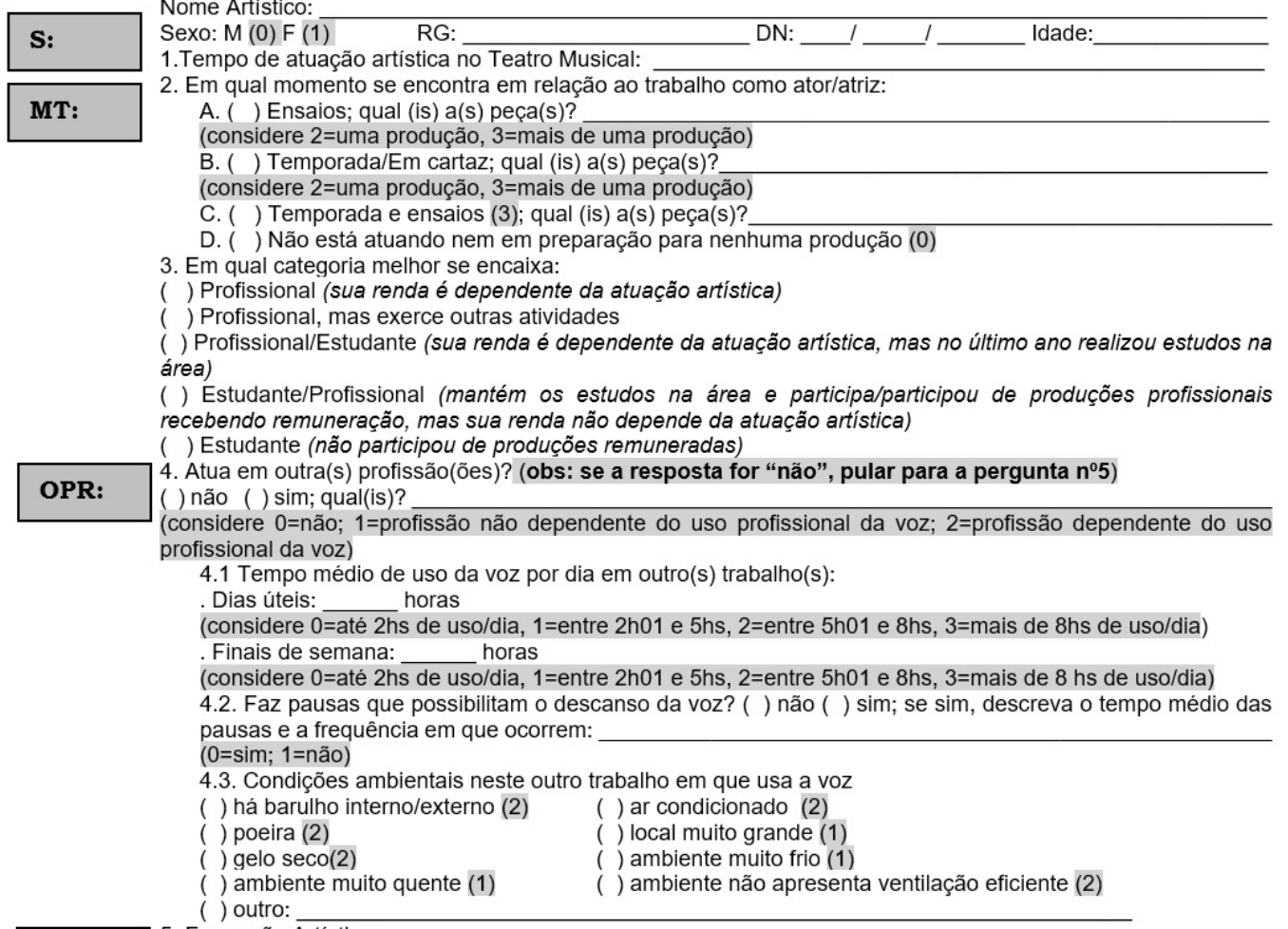

FA: 5. Formação Artística:

Como ocorreu/ocorre sua formação artística (todos os dados gerais mais relevantes à formação performática: teatro, teatro musical, dança, canto, etc.)

5.1 Durante sua formação teve estudos e/ou orientações sobre como ocorre a produção saudável da voz? ( ) não ( ) sim; E sobre bem-estar vocal? ( ) não ( ) sim Descreva algumas dessas orientações:

Segue tais orientações? ( ) não ( ) sim

(considere $0=$ orientações adequadas e são seguidas; $1=$ orientações adequadas ou inadequadas, mas não as segue; $2=$ orientações inadequadas, mas as segue; $3=$ não teve orientações)

6. Classificação vocal:

$\begin{array}{llll}\text { ( ) baixo ( ) baritono } & \text { ( ) tenor } & \text { ( ) contratenor ( ) contralto ( ) meio-soprano }\end{array}$

( ) soprano ( ) não sabe descrever ( ) outro:

7. Tipo de canto mais utilizado recentemente (últimos seis meses):

( ) Popula

$$
\begin{array}{ll}
\text { ( ) Belting / POP } & \text { ( ) MPB } \\
\text { ( ) Legit } & \text { ( ) Outro }
\end{array}
$$

( ) Erudito

$\begin{array}{ll}\text { ( ) Italiana } & \text { ( ) Inglesa } \\ \text { ( ) Francesa } & \text { ( ) Alemã }\end{array}$

Outra

AC:

8. Faz ou fez aula de canto? ( ) não ( ) sim; qual(is) estilo(s) estudado(s)? Descreva quais foram os profissionais que te acompanham/acompanharam e por quanto tempo:

(considere 0 =faz aula de canto, $1=$ fez aula de canto, mas a última foi há mais de seis meses, 2=não faz e nunca fez aula de canto)

AD:

9. Faz ou fez aula de dança? ( ) não ( ) sim; qual(is) o(s) estilo(s) estudados?

(considere 0 =faz aula de dança, 1 =fez aula de dança, mas a última foi há mais de seis meses, 2=não faz e nunca fez aula)

9.1. Durante a realização das coreografias o corpo assume posturas extremas em momentos de uso vocal? ( ) não ( ) sim; explique (se possivel, demonstre):

Percebe que alguma postura corporal interfere na produção da voz? ( ) não ( ) sim; explique:

(considere 0 =tendência positiva, $1=$ tendência negativa)

9.2. Após a realização das coreografias percebe aumento da tensão corporal?

( ) não ( ) sim; percebe que há alteração na produção vocal?

(considere 0=não há alteração, 1=percebe pouca alteração, 2=percebe grande alteração)

PF: 10. Faz ou fez alguma preparação física?

( ) não ( ) sim; qual?

(0=sim; 1 =não)

11. Faz ou fez aulas de teatro/interpretação? não ( ) $\operatorname{sim}(\quad)$

Descreva quais os profissionais que te acompanham/acompanharam e por quanto tempo: 
(considere $0=$ =ensaio diriqido por profissional da voz; $2=$ =ensaio não diriqido por profissional da voz; $3=$ não ensaia) 12.1. Descreva frequência e número de horas:

(considere: $0=$ até $2 \mathrm{hs}$ de uso/dia, $1=$ entre $2 \mathrm{~h} 01$ e $5 \mathrm{hs}, 2=$ entre $5 \mathrm{~h} 01$ e $8 \mathrm{hs}, 3=$ mais de $8 \mathrm{hs}$ de uso/dia)

MIC:

13. Faz uso de microfone ou outro recurso de amplificação vocal durante os ensaios?

( ) não ( ) sim ( ) às vezes; descreva:

(considere $0=\operatorname{sim}, 1=$ às vezes, $2=$ não)

13.1. Faz uso de microfone ou outro recurso de amplificação vocal durante as apresentações?

( ) não ( ) sim ( ) às vezes; descreva:

(considere $0=\operatorname{sim}, 1=$ às vezes, $2=$ não)

13.2. Se faz uso em alguma das situações, teve orientações ou treinamento de como utilizar o equipamento? ( ) não ( ) sim; qual(is)?

(considere $0=$ orientações adequadas e são seguidas; $1=$ orientações adequadas ou inadequadas, mas não as segue; $2=$ orientações inadequadas, mas as segue ou não teve orientações)

\begin{tabular}{|l|}
\hline AMB: \\
\hline TR: \\
\hline DIF: \\
\hline FP: \\
\hline
\end{tabular}

14. Condições ambientais durante a atuação:
( ) há barulho interno/externo (2)
( ) poeira (2)
( ) ar condicionado (2)
) ambiente muito frio (1)
( ) local muito grande (1)
( ) ambiente muito quente (1)
( ) ambiente não apresenta ventilação eficiente (2)

()outro:

15. Tipo respiratório:

$\begin{array}{llll}\text { ( ) costodiafragmático ( ) inferior ( ) misto } & \text { ( ) superior }\end{array}$

) não sabe descrever (deve ser conferido pelo avaliador):

(considere $0=$ costodiafragmático; 1 =inferior; $2=$ misto; $3=$ superior)

16. Quanto à psicodinâmica vocal no teatro, recentemente teve que modular a voz para transmitir:
( ) animação
( ) tristeza ( ) raiva
( ) grito
( ) medo ( ) insanidade
( ) fragilidade
( ) sons de animais
( ) sussurro
( ) bêbado
( ) idade mais avançada
( ) idade mais jovem
( ) cansaço

) outros; quais?

17. Apresenta dificuldades para manter:

a mesma qualidade vocal do início ao fim da apresentação/ensaio (diário)?

( ) nunca ( ) às vezes ( ) sempre; se assinalou "às vezes" ou "sempre", descreva:

(considere $0=$ =não, 1 =às vezes, $2=$ sempre)

a mesma qualidade vocal do início ao fim da semana?

( ) nunca ( ) às vezes ( ) sempre; se assinalou "às vezes" ou "sempre", descreva:

considere $0=$ não; 1 =às vezes; $2=$ sempre

18. Qual sua função dentro da produção?

( ) PROTAGONISTA - responsável por um papel de peso na trama (2)

( ) ENSEMBLE - somente integrante do coro cênico (1)

( ) SWING - substitui personagens do ensemble (2)

( ) DANCE CAPTAIN - bailarino responsável pela manutenção das coreografias. (0)

( ) COVER - tem um papel mais curto e substitui outros atores principais em seus papeis (1)

( ) ALTERNANTE - alterna um papel (geralmente principal) (1)

( ) PERSONAGEM SECUNDÁRIO (1)

( ) PIT SINGER - cantor que não entra em cena $(0)$

) STANDIN - não tem um papel na peça, mas pode entrar caso seja necessário (1)

Obs: se o participante estiver em mais de uma produção deve-se somar a pontuação das funções

19. Em relação à qualidade vocal, indique na escala abaixo, com um traço vertical, o quanto sua voz Ihe desagrada antes da prática artística diária:
Agrada
Desagrada

20. Em relação à qualidade vocal, indique na escala abaixo, com um traço vertical, o quanto sua voz lhe desagrada depois da prática artística:

Agrada

Desagrada

RSTR:

21. Em relação ao possível estresse proporcionado pela rotina da produção (ensaios, apresentações e etc.) indique na escala abaixo, com um traço vertical, o quanto considera estressante a sua rotina:

Nada Estressante

22. Realiza aquecimento e/ou desaquecimento da voz?

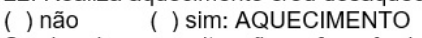

Se sim, descreva situações e frequência:

( ) sim: DESAQUECIMENTO ( ) sim: AMBOS

(considere $0=$ sim, aquecimento e desaquecimento; 2=só aquecimento ou só desaquecimento; 3=não faz nem aquecimento nem desaquecimento)

22.1 Procedimentos:

(considere 0 =procedimentos adequados; $1=$ parcialmente adequados $2=$ procedimentos inadequados

UV:

23. Tempo médio de uso da voz por dia:

23.1. dias úteis: trabalha horas e usa a voz por horas

(considere $0=$ até $2 \mathrm{hs}$ de uso/dia, $1=$ entre $2 \mathrm{~h} 01$ e $5 \mathrm{hs}, 2=\overline{=} \overline{\mathrm{entre}} 5 \mathrm{~h} 01$ e $8 \mathrm{hs}, 3=$ mais de $8 \mathrm{hs}$ de uso/dia)

23.2. finais de semana: trabalha __ horas e usa a voz por ___ horas

(considere $0=$ até $2 \mathrm{hs}$ de uso/dia, $\overline{1=e n t r e} 2 \mathrm{~h} 01$ e $5 \mathrm{hs}, 2=$ entre $5 \mathrm{h01}$ e $8 \mathrm{hs}, 3=$ mais de $8 \mathrm{hs}$ de uso/dia)

23.3. faz pausas que possibilitam o descanso da voz? ( ) não ( ) sim

(considere $0=\operatorname{sim} ; 1=$ não)

Se sim, descreva tempo médio das pausas e frequência em que ocorrem: 
FU: 24. Em relação ao fumo:

( ) nunca fumou

( ) fumou/fuma apenas durante atuação/ensaios; descreva a frequência e duração

( ) é ex-fumante; descreva por quanto tempo fumou; há quanto tempo parou; número médio de cigarros que fumava por dia:

( ) é fumante; descreva há quanto tempo fuma; número médio de cigarros por dia:

(considere $0=$ não fumantes ou ex-fumantes há 10 anos ou mais; $1=e x$-fumante há menos de 10 anos; $2=$ fumo em cena ou ensaios; $3=$ fumante)

25. Ingere bebida alcoólica?

( ) não ( ) sim; descreva: tipo de bebida; quantidade e frequência:

(considere $0=$ não, $1=\operatorname{sim}$ )

DR: 26. Usa ou usou drogas?

( ) não ( ) sim; descreva tipo, quantidade e frequência: (considere $0=$ não, $1=$ sim)

PR: 27. Usa prótese dentária?

( ) não ( ) sim; descreva tipo e há quanto tempo (considerar se precisa usar e não usa):

se sim, tem alguma queixa em relação ao uso dessa prótese?

( ) não ( ) sim; descreva:

considere $0=$ não usa e não precisa; 1=usa e tem queixa em relação ao uso; 2=precisa usar, mas não usa)

ó para mulheres:

( ) apresenta sintomas de tensão pré-menstrual? ( ) não （ ) sim; se sim, descreva:

( ) está grávida? ( ) não ( ) sim; se sim, tempo da gestação:

( ) encontra-se em período de menopausa? ( ) não ( ) sim; se sim, há quanto tempo:

( ) apresenta problemas hormonais? ( ) não ( ) sim; se sim, descreva: (considere para cada item: $0=$ não, $1=\operatorname{sim}$ )

SSE: 29. Assinalar a frequência em que ocorrem os itens abaixo:

(indique: 3 - diariamente/sempre; 2 - semanalmente/quase sempre; 1 - mensalmente/às vezes; 0 - nunca)

(3) (2) (1) (0) Frustração por conta da produção vocal

(3) (2) (1) (0) Perda do controle do padrão respiratório durante a fala habitual

(3) (2) (1) (0) Perda do controle do padrão respiratório durante o canto

(3) (2) (1) (0) Perda do controle do padrão respiratório durante a atuação

(3) (2) (1) (0) Perda do controle do padrão respiratório durante a dança

(3) (2) (1) (0) Perda do controle técnico da voz cantada

(3) (2) (1) (0) Perda do controle da dinâmica vocal

(3) (2) (1) (0) Perda do controle da frequência vocal

(3) (2) (1) (0) Fadiga Vocal pós-aquecimento

(3) (2) (1) (0) Fadiga vocal após apresentação

(3) (2) (1) (0) Necessidade de se ausentar da apresentação/ensaio devido à voz

(3) (2) (1) (0) Descontrole da afinação

(3) (2) (1) (0) Afonia

30. Outros comentários relevantes
ESCORE PRRD-TM:

Data: I
ESCORE TOTAL (PRRD-G + PRRD-TM):

Fonoaudióloga(o) 